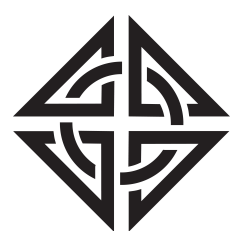

SCIENTIA
Sharif University of Technology

Scientia Iranica

Transactions F: Nanotechnology

www.scientiairanica.com

\title{
pH-responsive nanostructured polyaniline capsules for self-healing corrosion protection: The influence of capsule concentration
}

\author{
N. Pirhady Tavandashti ${ }^{a}$, M. Ghorbani ${ }^{a, b, *}$, A. Shojaei ${ }^{a, c, *}$, \\ J.M.C. Mol $^{\text {d }}$, H. Terryn ${ }^{\text {d,e }}$ and Y. Gonzalez-Garcia ${ }^{d}$ \\ a. Institute for Nanoscience and Nanotechnology (INST), Sharif University of Technology, Tehran, P.O. Box 11155-8639, Iran. \\ b. Department of Materials Science and Engineering, Sharif University of Technology, Tehran, P.O. Box 11155-9466, Iran. \\ c. Department of Chemical and Petroleum Engineering, Sharif University of Technology, Tehran, P.O. Box 11155-9465, Iran. \\ d. Department of Materials Science and Engineering, Delft University of Technology, 2628CD, Delft, The Netherlands. \\ e. Group of Electrochemical and Surface Engineering, Vrije Universiteit Brussel, B-1050, Brussels, Belgium.
}

Received 8 August 2016; received in revised form 18 January 2017; accepted 22 April 2017

\section{KEYWORDS}

Nanostructured polyaniline capsules;

MBT corrosion

inhibitor;

Organic coating;

Corrosion protection.

\begin{abstract}
Nanostructured hollow polyaniline (PANI) capsules are good candidates for encapsulation of corrosion inhibitors and $\mathrm{pH}$-responsive release when incorporated into organic coatings. In previous studies, the corrosion protection performance of PANI capsules, containing organic inhibitor 2-Mercaptobenzothiazole (MBT), was demonstrated. The present work studies the influence of capsule concentrations (i.e., 0.3, 1, and $2 \mathrm{wt} \%$ ) on the corrosion protection properties of the coating system. Anti-corrosion properties of different coatings were compared by means of Electrochemical Impedance Spectroscopy (EIS) and Scanning Vibrating Electrode Technique (SVET). MBT loaded PANI capsules in epoxy ester coating on AA2024-T3 substrate allow for a self-healing effect to be obtained during the corrosion process. The results showed that the concentration of MBT loaded PANI capsules greatly influences the corrosion protection properties of the coatings, and the best corrosion protection performance was observed for the coating system containing $1 \mathrm{wt} \%$ PANI capsules. The impedance value of the scratched area of this coating after 7 days of immersion was one order of magnitude higher than that of the control sample.

(C) 2017 Sharif University of Technology. All rights reserved.
\end{abstract}

\section{Introduction}

Due to the severe impact of corrosion on economy, industry, and environment, using strategies to prevent or slow down the rate of corrosion is of great importance. Application of organic coatings is one of the most widespread techniques for corrosion protection of

*. Corresponding authors. Tel: +98 2166165219 E-mail addresses: Ghorbani@sharif.edu (M. Ghorbani); Akbar.shojaei@sharif.edu (A. Shojaei)

doi: $10.24200 /$ sci.2017.4426 the metallic substrates. Since various external factors can cause formation of pores and cracks in the coating through which water and corrosive species can diffuse and reach the metal surface; recently, considerable interest has been given to the use of corrosion protection systems that provide not only a physical barrier against aggressive species, but also an active self-healing ability [1-4]. One of the main strategies to achieve the active effect is to apply micro/nanostructured hosting systems for encapsulation of corrosion inhibitors and to incorporate the capsules into the coating system. Accordingly, the micro/nanostructured capsules will store the inhibitors, and when corrosion starts (in case 
of degradation or damage in the coating), the capsules release the corrosion inhibitor to stop the corrosion process, providing a self-healing effect.

Conducting polymer micro/nanostructures has attracted much attention among researchers in recent years because of their unique intrinsic properties. Noticeably, the biggest advantage of nanostructured conducting polymers is that their structure and properties can be adjusted reversibly by doping-dedoping process [5], which can be controlled by adjusting electric potential or changing solution $\mathrm{pH}[6,7]$. Their response to chemical or electrical stimuli can make a change in their conductivity, volume, color, permeability, and hydrophilicity [5,7-10]. These characteristics make the conducting polymers fascinating candidates to be used as containers for encapsulation and smart release $[5,11-$ $13]$.

In our previous work [13], we studied the release of MBT from PANI capsules at different pHs (i.e., 1.5, 7, 11). The results from UV-vis and SERS analyses suggested that the encapsulation of MBT corrosion inhibitor in polyaniline hollow microspheres is an efficient way to achieve $\mathrm{pH}$-controlled release of the inhibitor as well as to avoid its undesirable leaching from the coating. It was observed that the release of MBT from PANI capsules is triggered at $\mathrm{pH} \sim 11$. The corrosion process is usually accompanied by $\mathrm{pH}$ changes in the local corrosion area; an increase in the local $\mathrm{pH}$ can take place during cathodic reaction of reduction of the dissolved oxygen $[14,15]$. Thus, the local increase of $\mathrm{pH}$ due to corrosion process can trigger the release of MBT from the capsules incorporated into the corrosion protective coatings, reducing the rate of corrosion significantly. The corrosion protection properties of MBT-loaded PANI capsules incorporated into epoxy ester coating were characterized. It was demonstrated that incorporation of MBT-loaded PANI capsules in the coating resulted in enhanced active protection performance in case of a coating defect, without compromising the barrier properties of the coating.

In the present work, the MBT corrosion inhibitor was encapsulated in PANI hollow capsules, and different concentrations of the capsules were embedded into epoxy ester coatings applied on AA2024 substrates. The anticorrosion properties of the coated samples and the effect of capsule concentration on the coating performance were investigated by adhesion pull-off tests, Scanning Vibrating Electrode Technique (SVET), and Electrochemical Impedance Spectroscopy (EIS) experiments.

\section{Experimental procedure}

\subsection{Materials}

Aniline $(\geq 99.0 \%)$, ammonium persulfate (APS, $\geq$
$98.0 \%$ ), sodium hydroxide ( $\geq 98.0 \%$, Pellets), hydrochloric acid (37\%), and Toluene (99.5\%) were bought from Merck Chemicals. $\beta$-naphthalene sulfonic acid ( $\beta$-NSA, $\geq 90.0 \%$ ) was acquired from Fluka Chemicals, and 2-Mercaptobenzothiazole (MBT) (97.0\%) was purchased from Sigma-Aldrich. All the chemicals were used as received.

\subsection{Fabrication of nanostructured PANI capsules and encapsulation of $M B T$}

Hollow microspheres of polyaniline (PANI) with nanosized shell were synthesized using a soft template method according to a procedure reported earlier [16]. The encapsulation of organic corrosion inhibitor MBT in PANI capsules was performed via stirring a mixture of PANI $(50 \mathrm{mg})$ and MBT $(0.3 \mathrm{~g})$ in ethanol solution $(10 \mathrm{~mL})$ at $500 \mathrm{rpm}$ for $3 \mathrm{~h}$. Then, the solution was evacuated using a vacuum pump along with stirring for $10 \mathrm{~min}$ in order to increase the amount of encapsulation. The capsules were washed and centrifuged for 3 times after the encapsulation procedure, followed by removing the supernatant and drying the capsules at room temperature overnight.

The loading of MBT in the capsules was investigated by UV-Visible Spectroscopy (PerkinElmer Lambda 950 instrument). The absorption at a wavelength of $324 \mathrm{~nm}$, which is attributed to the absorbance peak of MBT in ethanol, was monitored. The inhibitor-loaded PANI capsules were dispersed in ethanol. The dispersion was sonicated (UP400S, Hielscher Ultrasound Technology) for $20 \mathrm{~min}$ and centrifuged (6000 rpm, $30 \mathrm{~min})$. Then, the supernatant was studied via UV-vis spectroscopy. As a reference, ethanol solutions of MBT with identified concentrations were prepared and the calibration curve was made. The quantity of 2-mercaptobenzothiazole loaded into the hollow PANI microspheres is $1 \mathrm{wt} \%$.

\subsection{Coating preparation}

Aluminum AA2024 alloy was used as substrates for corrosion protection studies. Surface preparation for coating application was as follows: the substrates were ground up to 1200 grit paper, washed with detergent and distilled water, and degreased in acetone in ultrasound bath for $10 \mathrm{~min}$. Epoxy ester resin (EE430S, Rezitan) was selected for coating preparation.

The MBT loaded PANI capsules were dispersed in toluene and introduced into epoxy ester resin via sonication for $10 \mathrm{~min}$ to get a homogeneous suspension. The mixture was then applied using a Metrohm Autolab spin-coater (at $500 \mathrm{rpm}$ for $10 \mathrm{~s}$, then at $2000 \mathrm{rpm}$ for $20 \mathrm{~s}$ ) on AA2024 substrates. To investigate the effect of capsules concentration on the corrosion protection performance, the metallic substrates were coated with different coating systems containing $0.3,1$, and 2 wt $\%$ of MBT-loaded PANI-capsules. Moreover, 
pure epoxy ester coating (control) was also applied to AA2024 substrates for comparison. The mass loss on the curing of the samples, calculated by weight, was about $60 \%$. Using this value, the final compositions of different coating systems in dried coating after curing were estimated, as shown in Table 1. The dry film thickness of the coatings was $13.9 \pm 1.1 \mu \mathrm{m}$ measured by eddy current method (ED10 Eddy, Dual Scope MP40, Germany).

Pull-off tests, according to ASTM D4541, were conducted with a hydraulic pull-off tester (Elcometer, model HATE108) using a commercial 3M (M2000) adhesive cured for $24 \mathrm{~h}$ prior to testing.

\subsection{Corrosion protection evaluation}

In a typical EIS experiment, the coated substrates were scratched (scratch length $\approx 1 \mathrm{~mm}$ ) and placed into home-made cells, whereby an area of the sample with $8 \mathrm{~mm}$ diameter was exposed to $0.3 \mathrm{wt} \% \mathrm{NaCl}$ solution at room temperature for several days. A threeelectrode setup in a Faraday cage was used, and the impedance spectra were recorded at the open circuit potential. An $\mathrm{Ag} / \mathrm{AgCl}$ reference and a platinum counter electrode were immersed in the cell, and the metal substrate functioning as the working electrode was connected to an Impedance Analyzer (Solartron S1 1287). The current response was detected in the frequency range of $100 \mathrm{kHz}$ to $0.01 \mathrm{~Hz}$, and the applied voltage perturbation was $10 \mathrm{mV}$. Ten frequencies were assessed per decade. The spectra were obtained using the Zplot software and fitted with Zview. Constant Phase Elements (CPE) were used in all fittings instead of capacitances because of the deviation from the ideal capacitance behavior (the phase angle of capacitors was different from $-90^{\circ}$ ). The impedance of a $\mathrm{CPE}$ is defined as follows:

$$
Z_{\mathrm{CPE}}(\omega)=Q^{-1}(j \omega)^{-n}
$$

where $Z$ is the impedance, $Q$ is the admittance coefficient, $j^{2}=-1$ is the imaginary number, $\omega$ is the angular frequency ( $\left.\mathrm{rad} \mathrm{s}^{-1}\right)$, and $n$ is the $\mathrm{CPE}$ exponent. The following equation was used to calculate capacitance values for different elements in the equivalent circuit:

$$
C=Q\left(\omega_{\max }\right)^{n-1}
$$

where $\omega_{\max }$ is the frequency at which the imaginary impedance reaches a maximum for the respective time constant [17].

The scanning vibrating electrode technique (SVET, Applicable Electronics (USA)) was applied to analyze the anticorrosion properties of the coatings in 0.3 wt $\% \mathrm{NaCl}$ solution. The coating was scratched (scratch length $\approx 1 \mathrm{~mm}$ ) before the measurement to guarantee exposure of the metal to the aggressive solution. Then, the coated samples were sealed with adhesive tape and only the area $\left(\approx 3 \times 3 \mathrm{~mm}^{2}\right)$, including the scratch, was left uncovered. Measurements on areas with dimensions of $\sim 1 \times 2 \mathrm{~mm}^{2}$ were performed every $2 \mathrm{~h}$ during $24 \mathrm{~h}$, and the mean acquisition time per scan was $\sim 20 \mathrm{~min}$. The vibrating probe was a thin Pt needle, with a platinum black sphere of ca. $20 \mu \mathrm{m}$ in diameter deposited on its tip. The current density at $100 \mu \mathrm{m}$ above the sample surface was detected. The subsequent analysis of the SVET data was performed with the help of Quikgrid software. The repetition of SVET experiments showed a consistent trend in the results.

The surfaces of the scratched coatings after exposure to corrosive media were studied by JEOL JSMIT300 SEM.

\section{Results and discussions}

Nanostructured hollow PANI microspheres were synthesized by Pirhady Tavandashti et al. via a soft template method [16]. The diameters of the microspheres were mostly in the range of $1-2 \mu \mathrm{m}$, and they showed an average shell thickness of ca. $90 \mathrm{~nm}$. The FTIR and UV-vis spectroscopies [13,16] showed that the capsules have a backbone polymer structure of polyaniline in an emeraldine salt form.

The encapsulation of organic corrosion inhibitor 2-Mercaptobenzothiazole (MBT) was performed via stirring a mixture of PANI capsules and MBT in ethanol solution. UV-vis and SERS spectroscopies were used in the previous work [13] to confirm the encapsulation of MBT in PANI containers. In brief, after the encapsulation procedure, the capsules were washed and centrifuged for several times; then, the supernatant of washing of the capsules was studied using UV-vis spectroscopy. The absorption at a wavelength of $324 \mathrm{~nm}$ was monitored, which corresponds to the absorbance peak of MBT in ethanol. No peak related to the presence of MBT was observed in the supernatant of washing of the capsules. This was to eliminate the absorbed MBT on the surfaces of the capsules. To investigate the presence of MBT inside the polyaniline capsules, the capsules were broken by severe ultrasonication for $20 \mathrm{~min}$, and after centrifugation of the solution, the supernatant of broken capsules was studied by UV-vis spectroscopy. A strong absorption peak was detected in $324 \mathrm{~nm}$, confirming that MBT was successfully loaded in PANI capsules. To prove the encapsulation of MBT in PANI containers further, Surface Enhanced Raman Spectroscopy (SERS) was applied. The capsules were dispersed in water and a drop of dispersion was put on a SERS probe (which consisted of silver particles $(50-500 \mathrm{~nm})$ in a gelatin matrix on a glass substrate). When high laser power (i.e., $2.5 \mathrm{~mW}$ ) was applied to the capsules, which 
Table 1. Composition of different coating systems.

\begin{tabular}{cc}
\hline Sample & Description \\
\hline Control & Pure epoxy ester coating \\
Caps03 & $0.3 \mathrm{wt} \%$ of MBT loaded PANI capsules embedded into the coating \\
Caps1 & $1 \mathrm{wt} \%$ of MBT loaded PANI capsules embedded into the coating \\
Caps2 & $2 \mathrm{wt} \%$ of MBT loaded PANI capsules embedded into the coating \\
\hline
\end{tabular}

caused the capsules to burn off, the characteristic SERS signals for MBT were evidenced in the resulting spectrum. It showed that after the capsules were burnt out, MBT was released and absorbed on the surface of the SERS probe. Due to the surface enhancement effect of SERS probe, even the MBT encapsulated in single PANI capsules was detectable. This result also confirmed the encapsulation of MBT in the cavity of the capsules.

To investigate the effect of capsules concentration on the corrosion protection performance of the coatings, the metallic substrates were coated with different coating systems containing $0.3,1$, and $2 \mathrm{wt} \%$ of MBT loaded PANI capsules. Furthermore, pure epoxy ester coating (control) was applied on AA2024 substrates for comparison. The compositions of different coating systems and codes used during the investigation are shown in Table 1.

\subsection{Characterization of the coatings}

Since epoxy ester coating is transparent, there is a possibility to investigate the dispersion of hollow polyaniline capsules in the coating by optical microscopy (Figure 1). The optical microscopy images illustrated in Figure 1(a)-(d) were taken from the same area of the coating with a focus on different focal depths. It was observed that the PANI capsules were uniformly distributed in the coating matrix in three dimensions.

Besides barrier and active properties, the adhesion of the coatings is of great importance. Pull-off test was applied to the study of the adhesion of the coated samples. Figure 2 presents the results of pulloff tests, showing that the coatings, containing MBT loaded PANI capsules, have superior adhesion to that of pure epoxy ester coating (control). Moreover, the adhesion properties of the composite coatings significantly increased with increasing the concentration of PANI capsules in the coatings.

Visual examination of the surfaces of the sample and dolly (Table 2) revealed that by incorporation of PANI capsules $(\geq 1 \mathrm{wt} \%)$ in the coating system, the delamination of the coatings decreased. Furthermore, as the capsule content increases, the percentage of coating delamination decreases and the sample with $2 \mathrm{wt} \%$ of PANI capsules (Caps2) showed no signs of coating delamination with an adhesive fracture mode. These results suggest that PANI capsule content increases the adhesion strength of the epoxy ester coating.

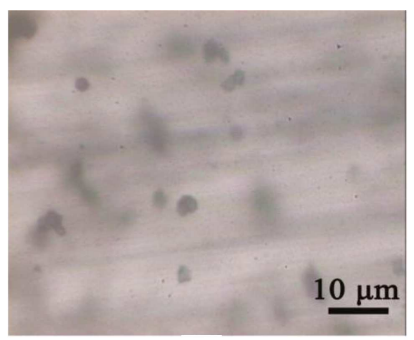

(a)

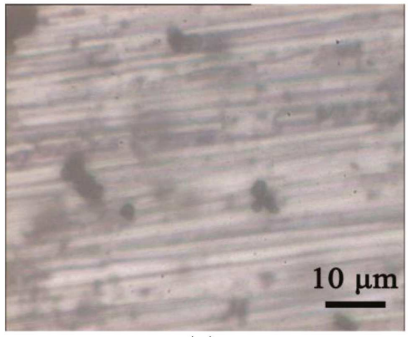

(c)

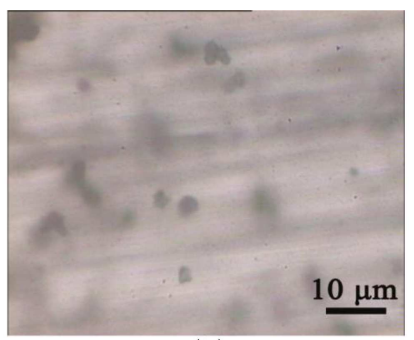

(b)

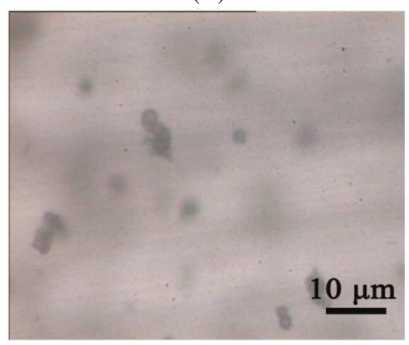

(d)
Figure 1. Optical microscopy of dispersion of MBT loaded PANI capsules in epoxy ester coating (Caps03) on AA2024 substrate: (a)-(d) The images were taken from the same area with focus on different focal depths.

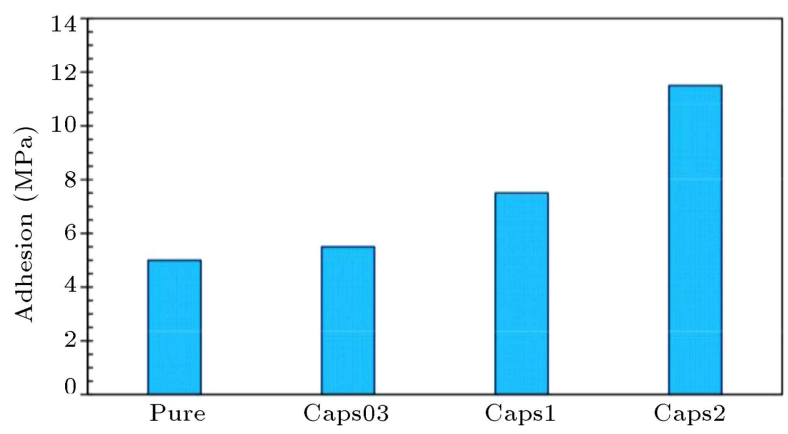

Figure 2. Pull-off adhesion test of different coating systems.

Table 2. Pull off delamination results.

\begin{tabular}{cc}
\hline Sample & Delamination (\%) \\
\hline Control & 80 \\
Caps03 & 90 \\
Caps1 & $\leq 1$ \\
Caps2 & 0 \\
\hline
\end{tabular}

\subsection{Evaluation of the corrosion protection of the coatings}

To study the self-healing properties of the coatings, EIS and SVET measurements were performed on the samples immersed in $0.3 \mathrm{wt} \% \mathrm{NaCl}$ solution. Prior to 
corrosion tests, scratches with $1 \mathrm{~mm}$ length were made in the coatings using a scalpel in order to induce the corrosion process.

\subsubsection{EIS measurements}

EIS measurements were performed on the scratched samples in $0.3 \mathrm{wt} \% \mathrm{NaCl}$ solution to evaluate the corrosion protection properties of the coated samples. In Figures 3 and 4, Bode plots of the scratched samples are shown after 1 and 7 days of immersion, respectively. After 1 day of immersion (Figure 3 ), the sample containing $1 \mathrm{wt} \%$ MBT-loaded PANI capsules (Caps1) presented higher impedance values than those of the other coating systems. The lowest impedance values were observed for Caps03 and control coating systems. After 7 days of immersion in $0.3 \mathrm{wt} \% \mathrm{NaCl}$ solution (Figure 4), Caps1 coating system showed still superior corrosion protection compared to other samples. It presented the highest impedance values amongst other coating systems. Although Caps03 and Caps2 coating systems showed poor protection performances with respect to sample Caps1, they still had higher impedance values than those of the control.

EIS results for all three coating systems showed three time constants corresponding to capacitance of the coating, oxide layer of bare metal, and corrosion processes in the scratch [13]. EIS spectra were fitted using the equivalent circuit presented in Figure 5. In this equivalent circuit, $R_{\text {sol }}$ is the solution resistance; $R_{\text {coat }}$ and $\mathrm{CPE}_{\text {coat }}$ are the resistance and capacitance
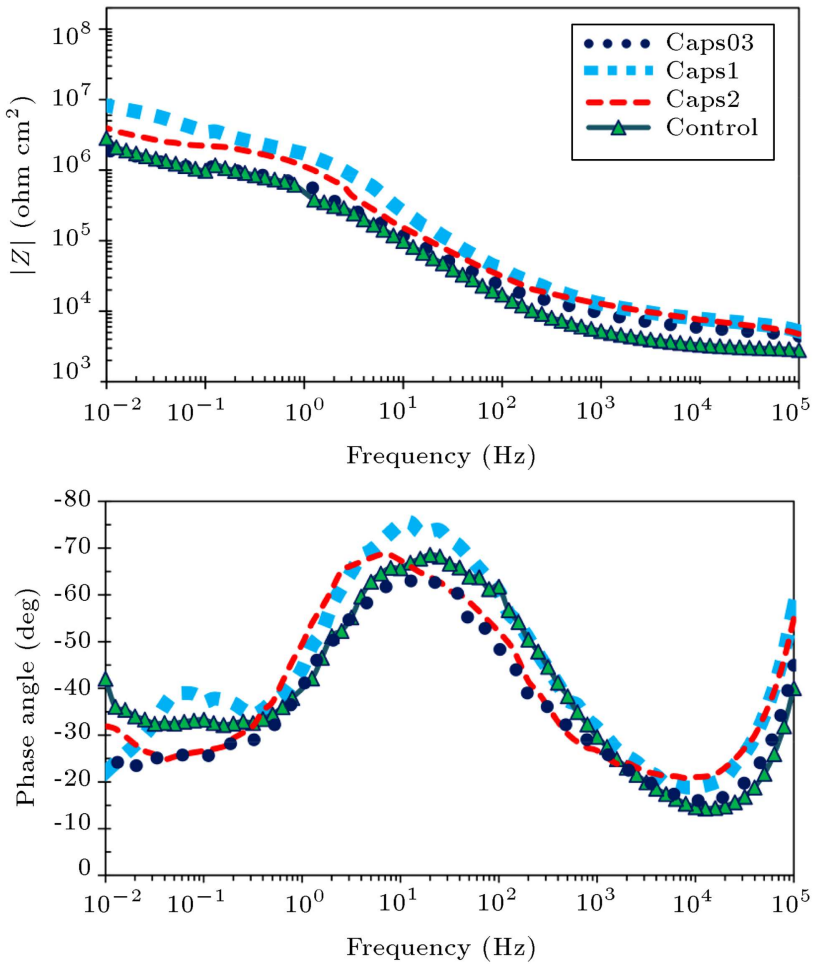

Figure 3. Bode plots of the scratched samples after 1 day of immersion in $0.3 \mathrm{wt} \% \mathrm{NaCl}$ solution.
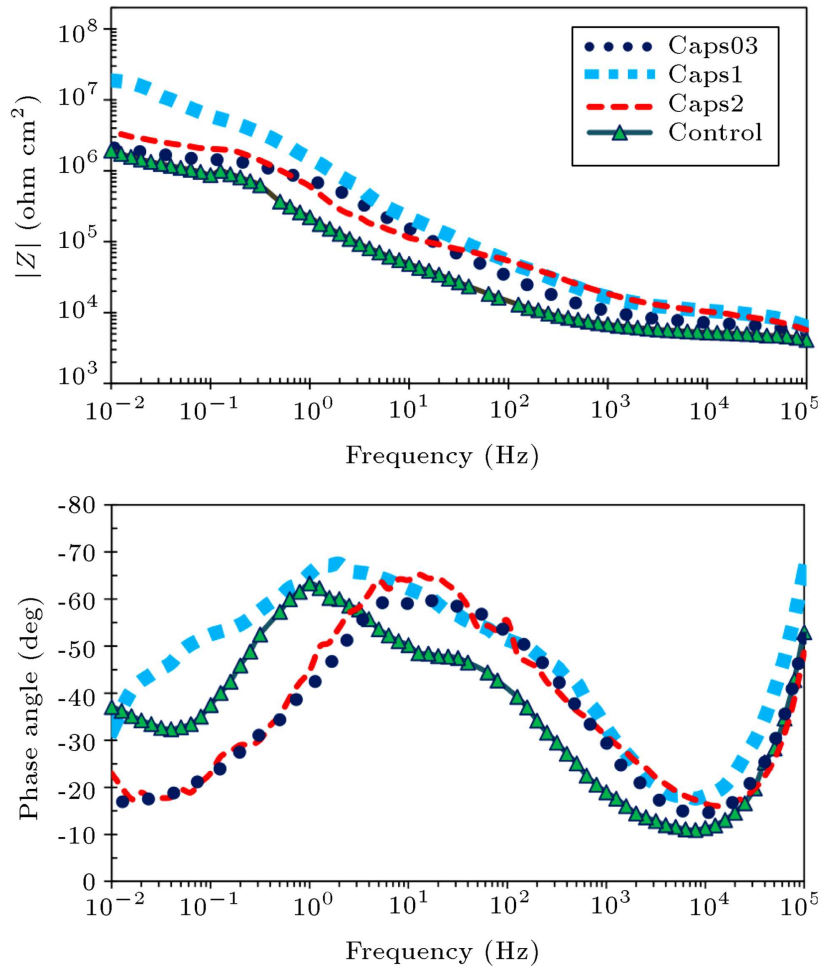

Figure 4. Bode plots of the scratched samples after 7 days of immersion in $0.3 \mathrm{wt} \% \mathrm{NaCl}$ solution.

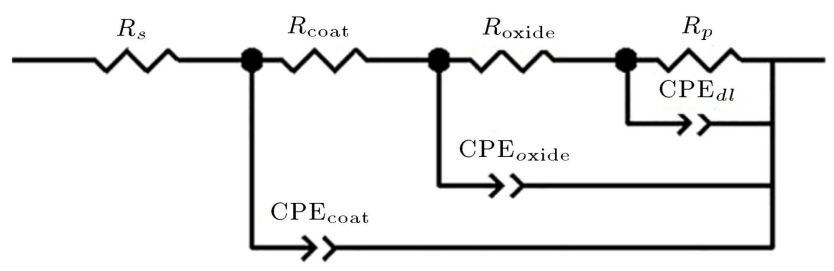

Figure 5. The equivalent circuit used for numerical fittings of EIS data.

of the coating, respectively; $R_{\text {oxide }}$ is the resistance of oxide layer and $\mathrm{CPE}_{\text {oxide }}$ is the capacitance of oxide layer; $R_{p}$ and $\mathrm{CPE}_{d l}$ are the polarization resistance and double-layer capacitance, respectively.

Figure 6(a) and (b) show the evolution of the capacitance and resistance of the oxide layer for the different scratched coatings during immersion in 0.3 wt $\% \mathrm{NaCl}$ solution. In Figure 6(a), the control sample showed higher capacitance values than the systems containing MBT-loaded PANI capsules. The systems with MBT-loaded PANI capsules presented low, similar and stable capacitance values during 7 days of immersion. This result indicates degradation in the oxide layer of the control coating with time. On the contrary, a more compact oxide layer was formed in the coatings containing MBT loaded PANI capsules. The evolution of the resistance of the oxide

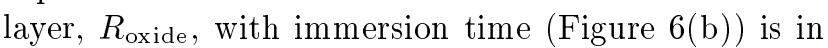
agreement with the capacitance behavior. The control (pure epoxy ester coating) shows the smallest values 


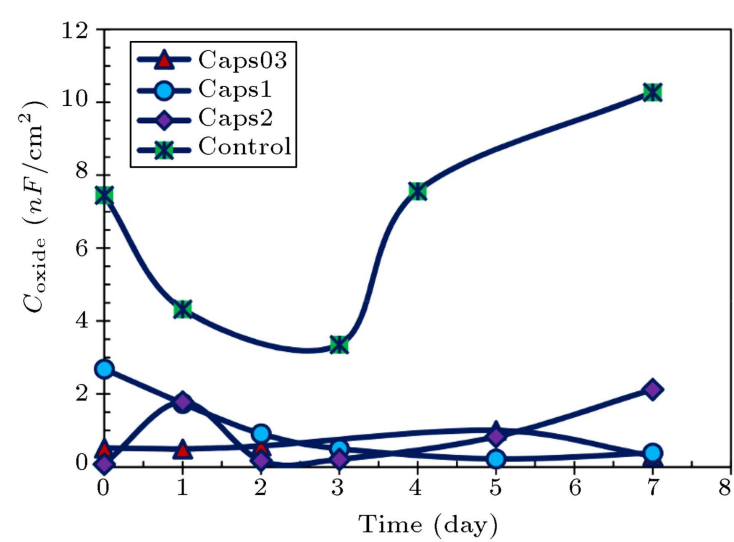

(a)

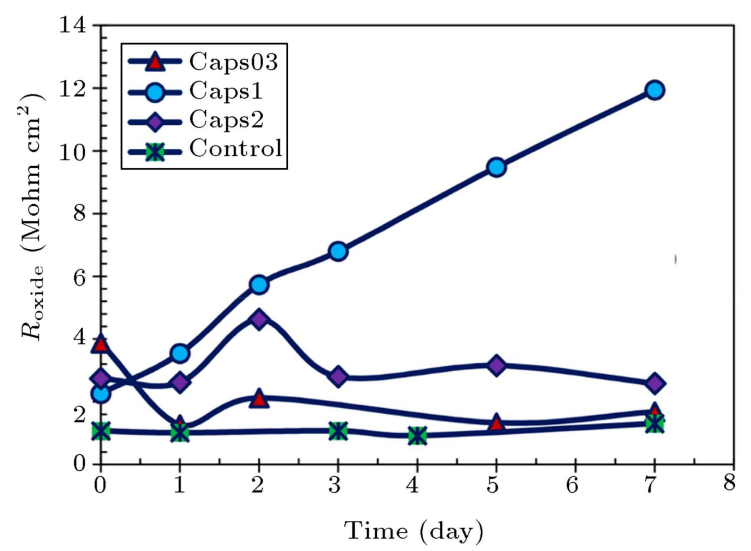

(b)

Figure 6. Evolution of (a) capacitance and (b) resistance of oxide layer for different coating systems during immersion in $0.3 \mathrm{wt} \% \mathrm{NaCl}$ solution.

of $R_{\text {oxide }}$ among other coating systems, confirming that the control coating has the weakest oxide layer among other systems. Nevertheless, it is important to note that the concentration of inhibitor-loaded PANI capsules in the coating plays an important role. The largest resistance values of oxide layer among all coating systems and during the total immersion period were measured for the coating containing $1 \mathrm{wt} \%$ MBT-loaded PANI capsules (Caps1). The resistance increases with immersion time reaching one order of magnitude higher value than that of the control sample after 7 days. This behavior indicates that the selfhealing of the defect area in the coating containing MBT loaded PANI capsules, is taking place. Systems Caps03 and Caps2 showed poorer self-healing behavior. The resistance values of oxide layer for Caps2 coating system were similar to those of Caps1 during the first 2 days of immersion. However, after 2 days, $R_{\text {oxide }}$ of Caps2 declined. For the case of Caps03, the resistance values of oxide layer were only slightly higher than that of the control sample. This result indicates that MBT-loaded capsule content in this coating was not sufficient to make a great difference from pure epoxy ester coating.

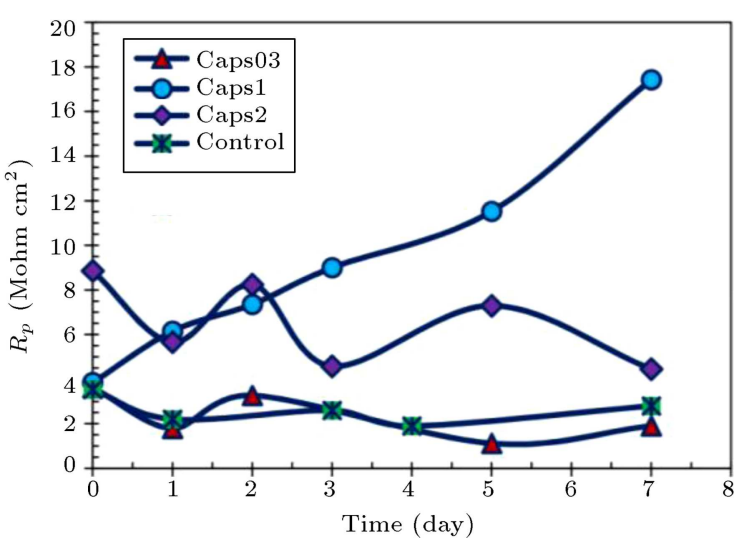

Figure 7. Evolution of polarization resistance for different coating systems during immersion in $0.3 \mathrm{wt} \%$ $\mathrm{NaCl}$ solution.

The evolution of polarization resistance for different scratched coatings during immersion in $0.3 \mathrm{wt} \%$ $\mathrm{NaCl}$ solution is shown in Figure 7. It was observed that $R_{p}$ shows similar trend to the resistance of the oxide layer (Figure 6(b)). Control coating has the smallest values of polarization resistance among other coating systems, meaning that this coating has the highest corrosion activity. Similarly, Caps03 coating system has approximately comparable $R_{p}$ values to those of the control coating. The largest values of $R_{p}$ for the coating, containing $1 \mathrm{wt} \%$ MBT loaded PANI capsules (Caps1), confirm superior corrosion protection properties of this coating system.

In our previous work, it was observed that the release of MBT from PANI capsules is triggered at $\mathrm{pH} 11$ [13]. During corrosion in neutral aqueous solutions, an increase in the local $\mathrm{pH}$ might take place through the reduction of the dissolved oxygen (1) $[14,15]$ :

$$
\mathrm{O}_{2}+2 \mathrm{H}_{2} \mathrm{O}+4 e^{-} \rightarrow 4 \mathrm{OH}^{-} .
$$

Therefore, the local increase of $\mathrm{pH}$ due to corrosion process is a trigger for MBT-loaded PANI capsules incorporated in the coatings to release the corrosion inhibitor. The released MBT forms a protective layer on the attacked metal surface, reducing the rate of corrosion significantly and providing a self-healing effect. However, EIS results show that the concentration of MBT-loaded capsules plays an important role in the self-healing properties of the coating. If it is more than an optimum amount, it can be even detrimental to the corrosion protection performance.

Figure 8 shows the SEM images of the scratched coating systems after 4 days of exposure to $0.3 \mathrm{wt} \%$ $\mathrm{NaCl}$ solution. Good agreement is observed between these SEM results, and the EIS results are discussed above. Figure 8(a) demonstrates that, for the control coating system, large aggregates of corrosion products are formed in the vicinity of scratch, evidencing the 


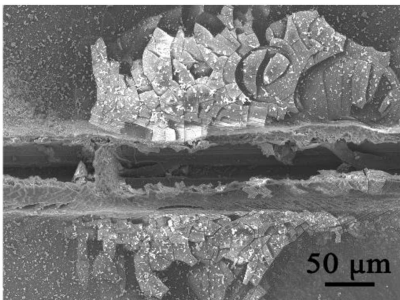

(a)

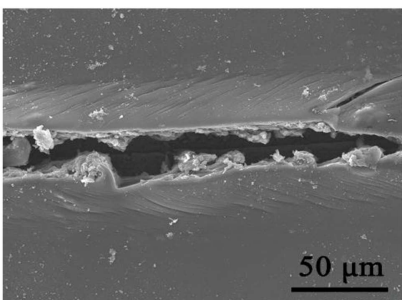

(c)

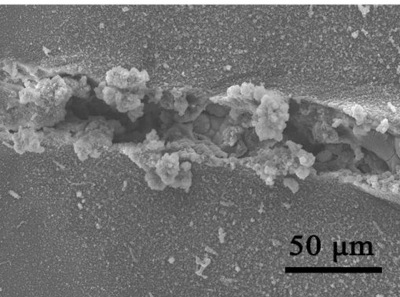

(b)

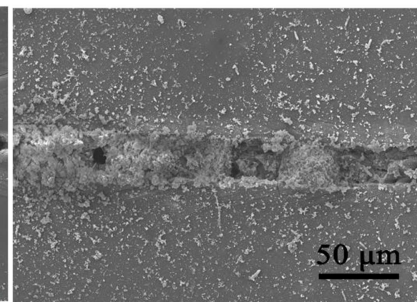

(d)
Figure 8. SEM images of the scratched coating systems: (a) Control, (b) Caps03, (c) Caps1 [13], and (d) Caps2 after 4 days of immersion in $0.3 \mathrm{wt} \% \mathrm{NaCl}$ solution.

high corrosion activity. Moreover, the presence of regions of undercoating corrosion was observed, and also delamination of the coating in some areas was detected for this coating system. For the coating systems containing MBT loaded PANI capsules (Figure 8(b)-(d)), no signs of delamination or undercoating corrosion were observed. However, the formation of the least corrosion products in the scratch area of Caps1 system confirms its superior corrosion protection and self-healing ability compared to other coating systems.

\subsubsection{SVET measurements}

The Scanning Vibrating Electrode Technique (SVET) was employed to assess the initial stages of corrosion development in the coatings and the self-healing performance. This technique can demonstrate localized corrosion activity by mapping the distribution of ca- thodic and anodic ionic currents along the surface. SVET maps of the coating defect area immersed in the $0.3 \mathrm{wt} \% \mathrm{NaCl}$ solution were obtained every two hours. The current density maps of the scratched coatings after $18 \mathrm{~h}$ immersion in $0.3 \mathrm{wt} \% \mathrm{NaCl}$ are presented in Figure 9(a)-(d). Figure 9(a) shows that the pure epoxy ester (control) coating indicates highly localized corrosion activity in the scratch area after $18 \mathrm{~h}$ immersion. For this coating, the defect was active during all tests, with a relatively increasing trend of anodic and cathodic current densities with immersion time. Figure 9(b)-(d) demonstrate that, in case of the epoxy ester coating containing MBTloaded PANI capsules, the corrosion current density is far less than that of the control coating, confirming the improvement of corrosion protection performance further in case of incorporation of PANI microspheres into the coating [13]. However, for this short immersion time, it was not possible to visualize obviously the differences between the coatings containing different concentrations of PANI capsules.

\section{Conclusions}

In this work, MBT corrosion inhibitor was encapsulated in PANI hollow microspheres, and the capsules were embedded into epoxy ester coatings applied to AA2024 substrates. The influence of capsules concentration on the corrosion protection performance of the coatings was evaluated. EIS and SVET results proved the superior corrosion protection of the coatings, containing MBT-loaded PANI capsules, in comparison to the pure epoxy ester coating. The results showed that the content of MBT loaded PANI capsules greatly influences the corrosion protection properties of the coating. The best corrosion protection performance was observed for the coating system containing 1 wt\% of PANI capsule (Caps1). However,

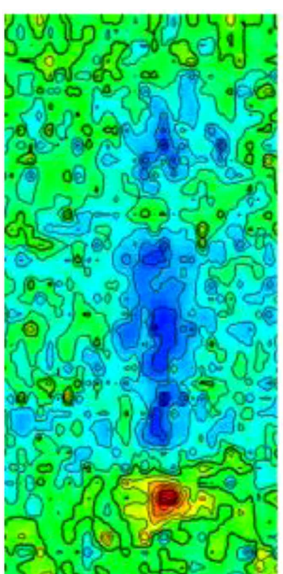

(a)

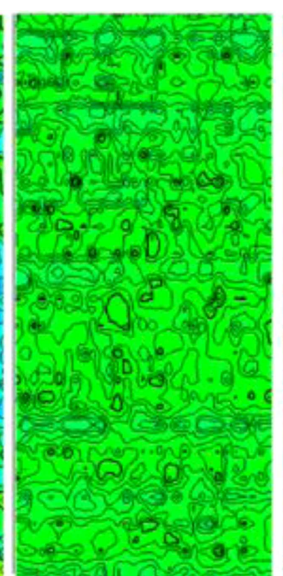

(b)

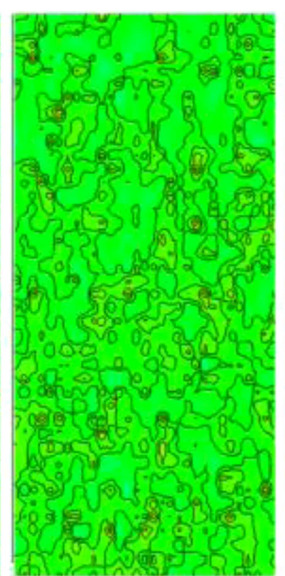

(c)

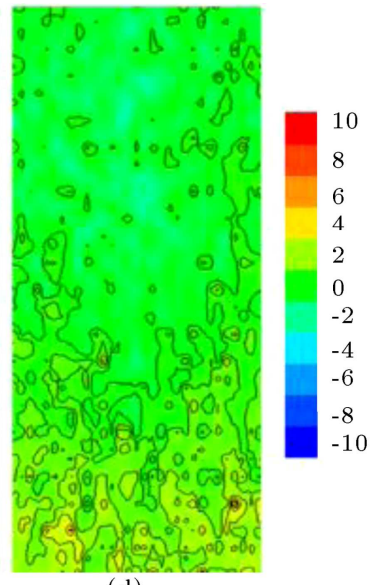

(d)

Figure 9. Current density maps of a scratch on (a) control, (b) Caps03, (c) Caps1, and (d) Caps2 coating systems after $18 \mathrm{~h}$ exposure to $0.3 \mathrm{wt} \% \mathrm{NaCl}$ solution. Scale units: $\mu \mathrm{A} \cdot \mathrm{cm}^{-2}$. Scanned area: $\approx 1 \mathrm{~mm} \times 2 \mathrm{~mm}$. 
incorporation of $0.3 \mathrm{wt} \%$ capsules (Caps03) into the coatings did not sufficiently improve the corrosion protection performance of epoxy ester coatings due to the lack of corrosion inhibiting materials. Extremely high concentration of PANI capsules (Caps02) in the coatings has also shown a negative effect, especially on immersion times longer than 2 days; it can be due to the increase of water uptake and/or decrease of the integrity of the coating by increase of PANI content.

\section{Acknowledgments}

The authors from Sharif University of Technology would like to express their appreciation to the Iran National Science Foundation for financial support.

\section{References}

1. Zheludkevich, M.L. Tedim, J. and Ferreira, M.G.S. "Smart coatings for active corrosion protection based on multi-functional micro and nanocontainers", Electrochim. Acta, 82, pp. 314-323 (2012).

2. González-García, Y., García, S.J., Hughes, A.E. and Mol, J.M.C. "A combined redox-competition and negative-feedback SECM study of self-healing anticorrosive coatings", Electrochem. Commun., 13, pp. 10941097 (2011).

3. González-Garcáa, Y., Mol, J.M.C., Muselle, T., Graeve, I.D., Van Assche, G., Scheltjens, G., Van Mele, B. and Terryn, H. "SECM study of defect repair in self-healing polymer coatings on metals", Electrochem. Commun., 13, pp. 169-173 (2011).

4. Pirhady Tavandashti, N., Ghorbani, M., Shojaei, A., Gonzalez-Garcia, Y., Terryn, H. and Mol, J.M.C. "pH responsive $\mathrm{Ce}(\mathrm{III})$ loaded polyaniline nanofibers for self-healing corrosion protection of AA2024-T3", Prog. Org. Coat., 99, pp. 197-209 (2016).

5. Abidian, M.R., Kim, D.H. and Martin, D.C. "Conducting-polymer nanotubes for controlled drug release", Adv. Mater., 18, pp. 405-409 (2006).

6. Michalska, A. and Maksymiuk, K. "Counter-ion influence on polypyrrole potentiometric $\mathrm{pH}$ sensitivity", Microchim. Acta, 143, pp. 163-175 (2003).

7. Hwang, J.H. and Pyo, M. "pH-dependent mass and volume changes of polypyrrole/poly (styrene sulfonate)", Bull. Korean Chem. Soc., 27, pp. 2067-2070 (2006).

8. Chen, Q., Yu, X., Pei, Z., Yang, Y., Wei, Y. and Ji, Y. "Multi-stimuli responsive and multi-functional oligoaniline-modified vitrimers", Chem. Sci., 8, pp. 724-733 (2017).

9. Park, D.E., Choi, H.J. and Manh Vu, C. "Stimuli- responsive polyaniline coated silica microspheres and their electrorheology", Smart Mater. Struct., 25, pp. 1-11 (2016).

10. Sarauli, D., Borowski, A., Peters, K., Schulz, B., Fattakhova-Rohlfing, D., Leimkühler, S. and Lisdat, F. "Investigation of the pH-dependent impact of sulfonated polyaniline on bioelectrocatalytic activity of xanthine dehydrogenase", ACS Catal., 6, pp. 71527159 (2016).

11. Kepinska, D., Blanchard, G.J., Krysinski, P., Stolarski, J., Kijewska, K. and Mazur, M. "Pyrene-loaded polypyrrole microvessels", Langmuir, 27, pp. 1272012729 (2011).

12. Vimalanandan, A., Lv, L.P., Tran, T.H., Landfester, K., Crespy, D. and Rohwerder, M. "Redox-responsive self-healing for corrosion protection", Adv. Mater., 25, pp. 6980-6984 (2013).

13. Pirhady Tavandashti, N., Ghorbani, M., Shojaei, A., Mol, J.M.C., Terryn, H., Baert, K. and GonzalezGarcia, Y. "Inhibitor-loaded conducting polymer capsules for active corrosion protection of coating defects", Corr. Sci., 112, pp. 138-149 (2016).

14. Boag, A., Hughes, A.E., Glenn, A.M., Muster, T.H. and McCulloch, D. "Corrosion of AA2024-T3. Part I: localised corrosion of isolated IM particles", Corr. Sci., 53, pp. 17-26 (2011).

15. Glenn, A.M., Muster, T.H., Luo, C., Zhou, X., Thompson, G.E., Boag, A. and Hughes, A. "Corrosion of AA2024-T3 Part III: Propagation", Corr. Sci., 53, pp. $40-50$ (2011).

16. Pirhady Tavandashti, N., Ghorbani, M. and Shojaei, A. "Controlled growth of hollow polyaniline structures: from nanotubes to microspheres", Polym., 54, pp. 5586-5594 (2013).

17. Hsu, C.H. and Mansfeld, F. "Technical note: Concerning the conversion of the constant phase element parameter $Y_{0}$ into a capacitance", Corros., 57, pp. 0915 (2001).

\section{Biographies}

Nahid Pirhady Tavandashti received her PhD degree in Nanomaterials from Institute for Nanoscience and Nanotechnology (INST), Sharif University of Technology. She has worked on self-healing coatings from 2007, when she started her MSc project. Her research interests are stimuli-responsive micro/nanocontainers, synthesis of nanocapsules and nanoreservoirs, smart self-healing coatings, and conducting polymers.

Mohammad Ghorbani is a Full Professor at the Department of Material Science and Engineering at Sharif University of Technology in Tehran, Iran. He earned his Master (1986) and doctoral (1991) degrees from Manchester University, UMIST, UK. His research covers a variety of coatings including electrodeposition 
of metallic coatings, composite coatings, conversion coatings, and nano coatings, leading to publication of more than one hundred ISI papers.

Akbar Shojaei is a Professor at the Department of Chemical \& Petroleum Engineering of Sharif University of Technology. He received his PhD degree in Polymer Composite Processing from Amirkabir University of Technology in 2002. He joined Sharif University of Technology in 2003 as an Assistant Professor and initiated a focused research program on polymeric systems. Currently, his main research interests are to develop advanced functional and structural polymer nano/composites for various applications such as biomedical, smart/active surface coatings, food packaging, friction materials, and engineering load- bearing components. Particularly, processing, fabricating, and unearthing relationships between micro/molecular structure and macroscopic properties of polymer composites are under intensive investigation. Dr. Shojaei has published more than 70 refereed journal articles and given scientific talks in many international conferences.

J.M.C. Mol. His/her biography was not available at the time of publication.

H. Terryn. His/her biography was not available at the time of publication.

Y. Gonzalez-Garcia. His/her biography was not available at the time of publication. 